\title{
Louise Poulin
}

(1991)

\section{"Les relations \\ entre blancs et amérindiens: \\ une perspective historique. \\ Une entrevue avec Pierrette Désy.”}

Un document produit en version numérique par Jean-Marie Tremblay, bénévole, professeur de sociologie au Cégep de Chicoutimi

Courriel: jean-marie_tremblay@uqac.ca

Site web pédagogique : http://www.uqac.ca/jmt-sociologue/

Dans le cadre de: "Les classiques des sciences sociales" Une bibliothèque numérique fondée et dirigée par Jean-Marie Tremblay, professeur de sociologie au Cégep de Chicoutimi

Site web: http://classiques.uqac.ca/

Une collection développée en collaboration avec la Bibliothèque Paul-Émile-Boulet de l'Université du Québec à Chicoutimi

Site web: http://bibliotheque.uqac.ca/ 


\section{Politique d'utilisation de la bibliothèque des Classiques}

Toute reproduction et rediffusion de nos fichiers est interdite, même avec la mention de leur provenance, sans l'autorisation formelle, écrite, du fondateur des Classiques des sciences sociales, Jean-Marie Tremblay, sociologue.

Les fichiers des Classiques des sciences sociales ne peuvent sans autorisation formelle:

- être hébergés (en fichier ou page web, en totalité ou en partie) sur un serveur autre que celui des Classiques.

- servir de base de travail à un autre fichier modifié ensuite par tout autre moyen (couleur, police, mise en page, extraits, support, etc...),

Les fichiers (.html, .doc, .pdf., .rtf, .jpg, .gif) disponibles sur le site Les Classiques des sciences sociales sont la propriété des Classiques des sciences sociales, un organisme à but non lucratif composé exclusivement de bénévoles.

Ils sont disponibles pour une utilisation intellectuelle et personnelle et, en aucun cas, commerciale. Toute utilisation à des fins commerciales des fichiers sur ce site est strictement interdite et toute rediffusion est également strictement interdite.

L'accès à notre travail est libre et gratuit à tous les utilisateurs. C'est notre mission.

Jean-Marie Tremblay, sociologue

Fondateur et Président-directeur général, LES CLASSIQUES DES SCIENCES SOCIALES. 
Cette édition électronique a été réalisée par Jean-Marie Tremblay, bénévole, professeur de sociologie au Cégep de Chicoutimi à partir de :

Louise Poulin

“LES RELATIONS ENTRE BLANCS ET AMÉRINDIENS: UNE PERSPECTIVE HISTORIQUE. Une entrevue avec Pierrette Désy”.

Un article publié dans la revue Contact CIEC, no. 10, no 1, printemps 1991, pp. 9-12. Ottawa: Conseil international d'études canadiennes.

[Autorisation formelle accordée par Mme Désy le 29 juillet 2008 de diffuser cet article dans Les Classiques des sciences sociales.]

Courriel : desy.pierrette@uqam.ca

Polices de caractères utilisée :

Pour le texte: Times New Roman, 12 points.

Pour les citations : Times New Roman, 12 points.

Pour les notes de bas de page : Times New Roman, 12 points.

Édition électronique réalisée avec le traitement de textes Microsoft Word 2004 pour Macintosh.

Mise en page sur papier format : LETTRE (US letter), 8.5'’ x 11'’)

Édition numérique réalisée le 2 août 2008 à Chicoutimi, Ville de Saguenay, province de Québec, Canada. 
Louise Poulin

"Les relations entre blancs et amérindiens: une perspective historique. Une entrevue avec Pierrette Désy."

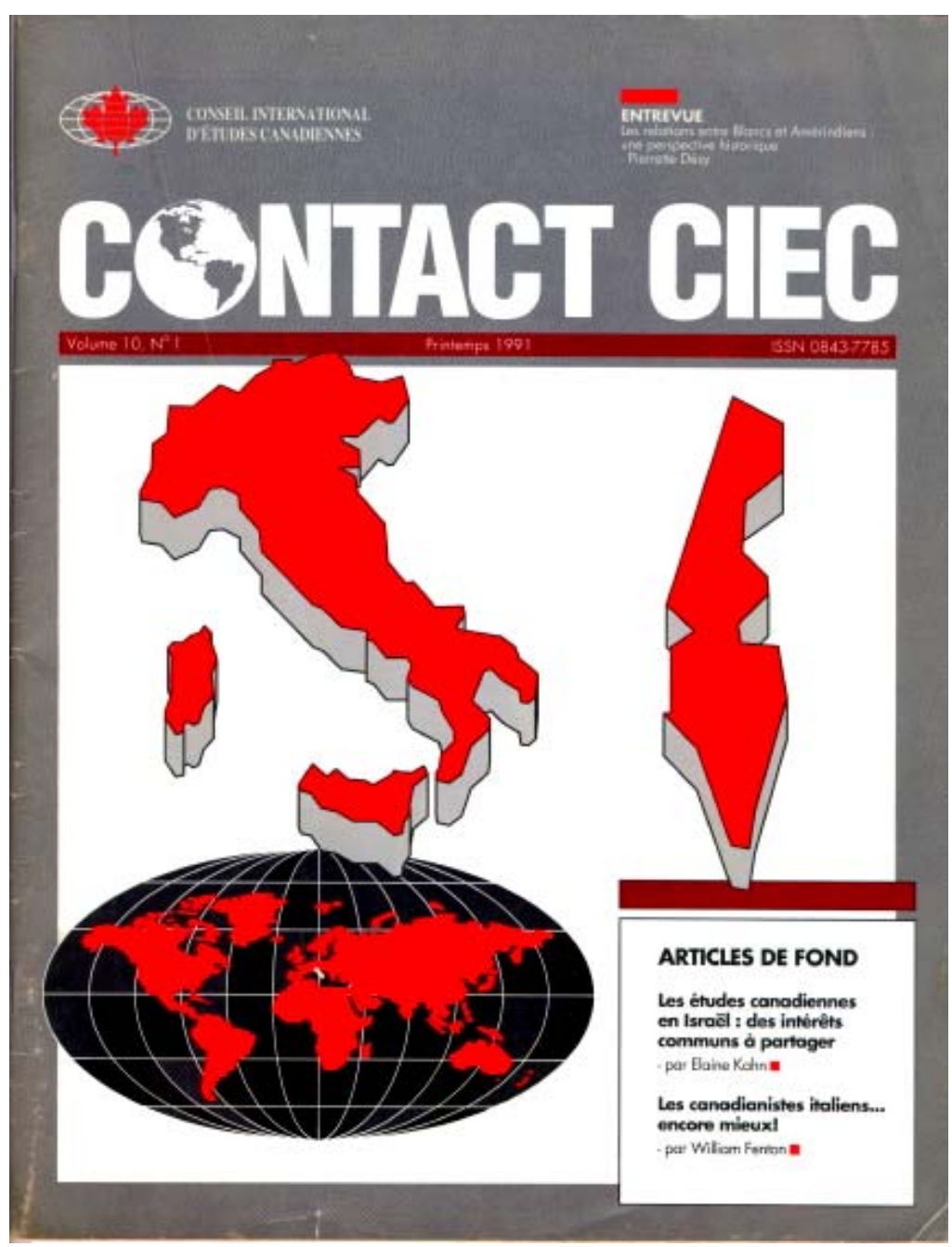

Un article publié dans la revue Contact CIEC, no. 10, no 1, printemps 1991, pp. 9-12. Ottawa: Conseil international d'études canadiennes. 


\section{Louise Poulin}

\section{"Les relations entre blancs et amérindiens: une perspective historique. Une entrevue avec Pierrette Désy”.}

Un article publié dans la revue Contact CIEC, no. 10, no 1, printemps 1991, pp. 9-12. Ottawa: Conseil international d'études canadiennes.

Pierrette Désy est professeur au Département d'histoire de l'Université du Québec à Montréal. Elle a fait des études d'ethnologie à Paris où elle a obtenu son doctorat de 3e cycle à la Sorbonne. Elle est spécialisée en anthropologie culturelle et en ethnohistoire; ses recherches portent sur les sociétés amérindiennes contemporaines et des siècles passés.

Auteur de nombreux articles, ses publications comprennent Trente ans de captivité chez les Indiens Ojibwa. Le récit de John Tanner 1 (Payot, 1983), elle a également contribué au Dictionnaire des Mythologies 2 (Flammarion, 1981), et au Dictionnaire des Poétiques ${ }^{3}$ (à paraître chez le même éditeur). Par ailleurs, elle a fait une recherche sur l'occupation territoriale des Amérindiens du Canada au XIXe siècle (vol. II de l'Atlas historique du Canada). Elle prépare présentement un manuscrit portant sur les captivités entre les Français et les Amérindiens au XVIle et XVIlle siècle. Elle a été présidente de la Société canadienne d'anthropologie en 1985-86.

Dans le cadre de la crise d'Oka/Kanesatake, Pierrette Désy a publié en août 1990 une série de trois articles portant sur les revendications historiques des Iroquois de Kanesatake et un article « ethnolittéraire » dans Culture en mai 1991.

1 Livre disponible dans Les Classiques des sciences sociales. JMT.

2 Texte disponible dans Les Classiques des sciences sociales. JMT.

3 Texte inédit, jamais publié, maintenant disponible, sous le titre "Ethnopoétique amérindienne” dans Les Classiques des sciences sociales. JMT. 
Elle a également fait une présentation à Kahnawake devant les délégués du Parlement européen en janvier 1991.

Elle espère un jour mettre sur pied un centre d'études autochtones afin de mieux faire connaître les peuples amérindiens et leurs cultures.

Nous avons interrogé Mme Désy, afin qu'elle nous aide à mieux comprendre les problèmes entre Blancs et Amérindiens ainsi que les enjeux. Elle nous fait part ici de ses idées.

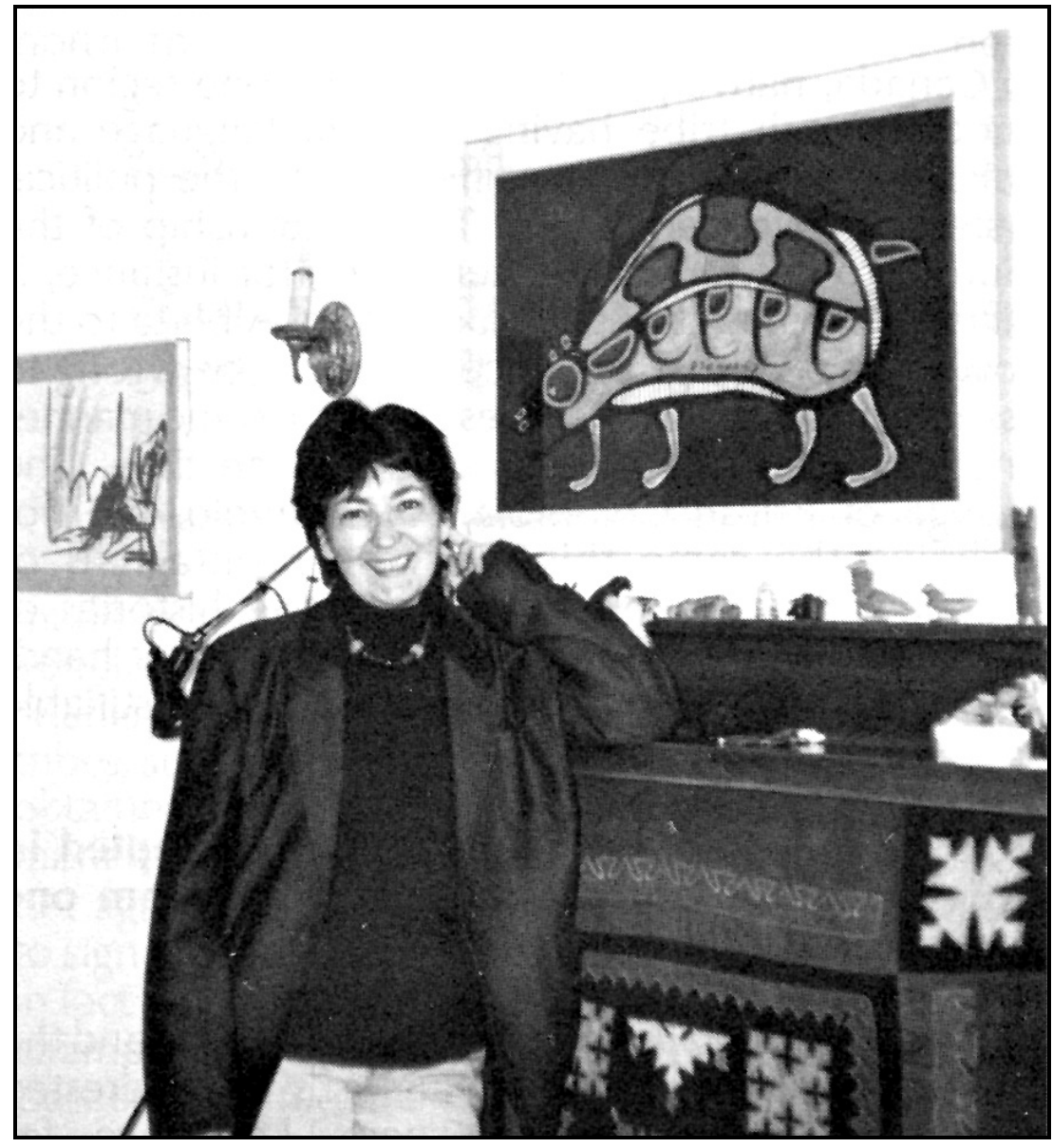

Pierrette Désy 
En tant que chercheuse comment la crise d'Oka de cet été vous a-t-elle marquée?

Je ne suis pas une spécialiste des peuples iroquois, pourtant dès le début de la crise, j'ai été frappée par le manque de connaissances des commentateurs et des journalistes. Le plus souvent, on racontait n'importe quoi. Il y avait un besoin urgent de comprendre la situation du point de vue historique. Dans les années 1970, j'ai eu l'occasion de suivre de près des mouvements autochtones comme celui de l'« American Indian Movement ». Il y avait entre le siège de Kanesatake, version 1990 et le siège de Wounded Knee, version 1973, des points communs. Au cours de mes recherches pour l'Atlas historique du Canada, j'avais recueilli une documentation pour toutes les réserves du Canada au XIXe siècle. Cela m'a donc été relativement facile à partir de mes dossiers sur les Iroquois et les Algonquins du lac des Deux-Montagnes, de faire l'historique des revendications et de montrer que depuis le début du XVIIIe siècle, les peuples revendiquaient systématiquement la même chose. C'est donc dire sans ironie aucune, que ce dossier aurait pu et aurait dû être réglé depuis longtemps. Plus généralement, la crise a permis aux Québécois et aux Canadiens de découvrir l'ampleur des revendications autochtones sans pour autant les connaître et nous faire connaître. Ce qui m'a frappée en outre, c'est le sentiment général de mauvaise conscience collective qui est monté comme un cri de la plupart des Canadiens et des Québécois. Mais en même temps, on peut se demander à quoi sert la mauvaise conscience si on ne peut pas régler les situations et offrir des solutions concrètes?

Quand on parle de revendications autochtones on peut les diviser en deux groupes, soit les revendications territoriales, soit les revendications d'autonomie. Sont-elles étroitement liées?

C'est évident qu'elles sont liées, on ne peut pas les séparer. C'est un peu comme si on donnait au Québec une autonomie sans territoire ou un territoire sans autonomie. On voit ce que ça donne aujourd'hui : beaucoup de problèmes. Pour les autochtones, il est extrêmement important qu'ils puissent avoir et un territoire et une autonomie a l'intérieur même de ce territoire. On voit par exemple avec les Sechelts en Colombie-Britannique, qui ont acquis une certaine forme d'autono- 
mie, comment ils peuvent manoeuvrer en ayant des bureaux et des commerces. Dans les années 1960 aux États-Unis, le Bureau of Indian Affairs voulait abolir les réserves et il avait réussi à convaincre un certain nombre de tribus dont les Menominee du Wisconsin de laisser leur réserve. Les Menominee avaient une réserve relativement grande ou se trouvait un moulin. Les gens de la réserve y travaillaient et l'économie de la réserve fonctionnait relativement bien grâce à un consentement commun. Mais du moment où la réserve des Menominee cessa d'exister, sans statut politique particulier, l'économie se désintégra peu à peu. Les Menominee risquaient alors de perdre leur sentiment d'appartenance culturelle et de se disperser. La réserve, le dernier rempart contre l'Autre, est donc très importante puisqu'elle a des frontières définies qui fait que l'Indien se retrouve dans son espace culturel. Le gouvernement américain a dû abandonner sa politique d'abolition des réserves parce que c'était une catastrophe. Quand Jean Chrétien, alors ministre des Affaires indiennes, présente son fameux "Livre blanc », un projet visant l'abolition des réserves, les Amérindiens lui répondent par le «Livre rouge » qui s'oppose au projet. La réserve est une forme d'autonomie. Il faudrait donc, en principe, accorder une autonomie plus grande dans un territoire plus grand. Mais ceci n'est pas une tâche facile puisque les autochtones ne revendiquent pas toujours la même chose.

D'un endroit à l'autre au Canada, les autochtones sont différents, avec leur propre langue et leur propre culture. Ils ont des relations différentes aux systèmes politiques qui les gouvernent. Le rapport des Hurons à l'État du Québec est différent de celui, par exemple, des Pieds-Noirs à celui de l'Alberta. Nous avons donc tort de toujours penser que tous les autochtones vont agir de la même façon et avoir les mêmes désirs au même moment. Les Nishga de la Colombie-Britannique ne revendiquent pas les mêmes choses que les Montagnais de la Côte nord. L'histoire est également différente pour ces peuples-là. Il y a donc des différences notables d'un groupe à l'autre. Par contre, tous les autochtones ont un désir légitime : celui de régler les revendications territoriales. 


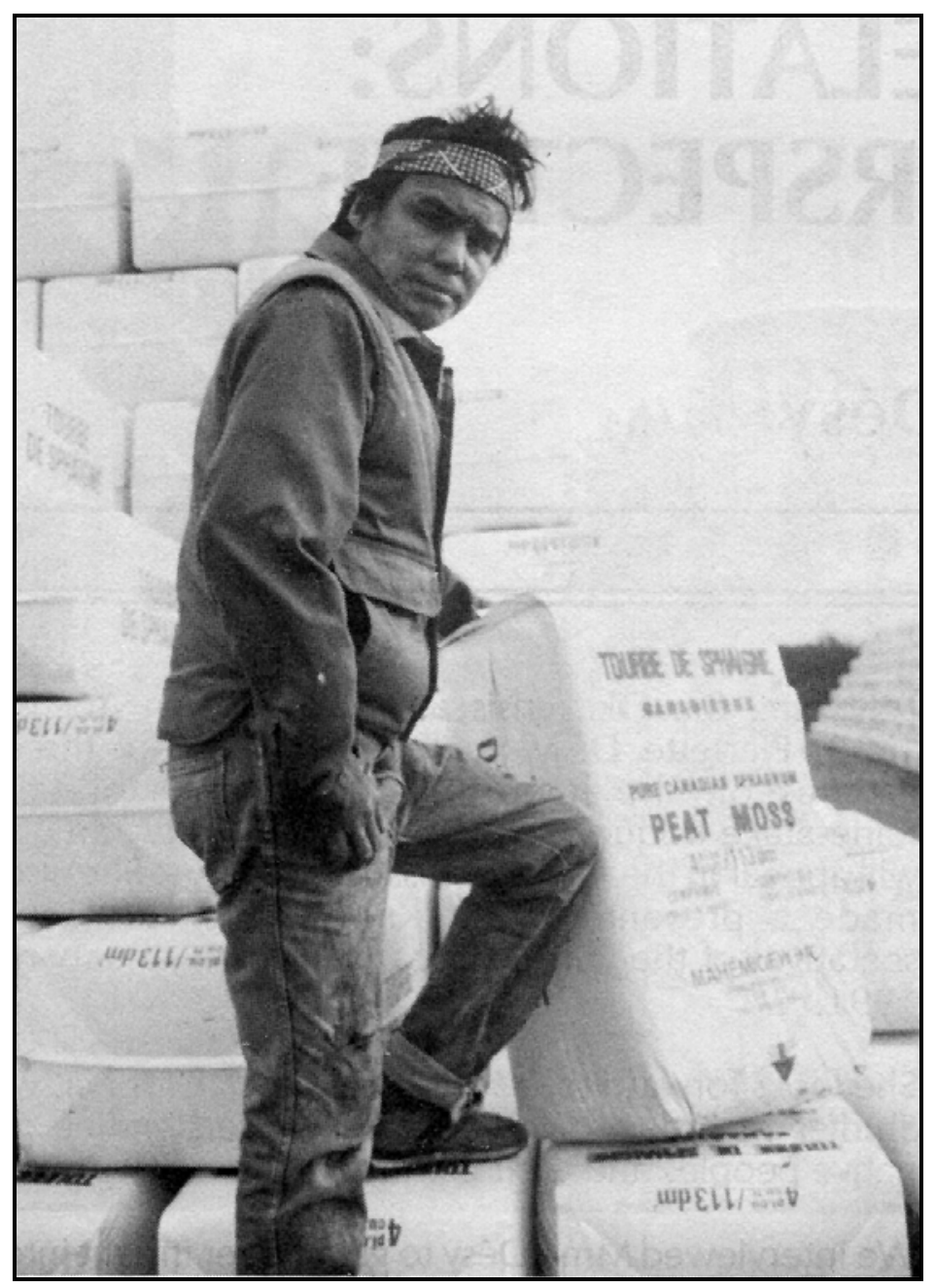

Ci-dessus : Travailleur amérindien à l'emploi de la compagnie Mahemigew Peat Moss, réserve de Lennox Island, Île-du-Prince-Édouard, 1985.

À votre avis, quel genre de politique devrait-on adopter pour régler ces revendications territoriales qui sont différentes d'une province à l'autre?

Idéalement, il faudrait écouter les gens qui ont quelque chose à dire, c'est-àdire d'abord les intéressés, et ensuite, les experts. Prenons le cas des Hurons du Québec : ils ont des revendications à propos de I'Île d'Orléans. Dans ce cas-là, il s'agit de mettre sur pied une équipe dont le but consiste à retrouver les documents historiques, cartographiques, etc. qui permettront aux Hurons de régler cette question. Dans la plupart des régions du Canada, on trouve effectivement des équipes 
de ce genre formées de chercheurs autochtones et non-autochtones. Observons que les revendications sont différentes les unes des autres : elles se suivent, mais ne se ressemblent pas nécessairement. Il faudrait repenser le ministère des Affaires indiennes qui ne répond pas efficacement à la demande. On dit qu'il y a 1500 revendications territoriales en suspens et qu'une seule est réglée par année. Il y a évidemment quelque chose qui ne tourne pas rond. Le ministère des Affaires indiennes pourrait se transformer en se consacrant uniquement aux règlements de revendications territoriales. Je suis consciente par contre que le problème ne se règle pas si facilement. Enfin, ce serait un début.

\section{Mais si les documents et les preuves existent, pourquoi est-ce si difficile de régler une revendication territoriale?}

Il y a deux types de revendications territoriales. La première concerne une seule nation qui a des revendications ponctuelles. On pense ici aux Hurons et aux Quarante Arpents qui faisaient partie de leur territoire et que le gouvernement a repris. C'est le genre de revendication qui est facilement réglable. Les revendications du deuxième type, plus nombreuses, sont beaucoup plus complexes. Elles se situent au plan de règlements territoriaux qui doivent prendre en considération les " glissements progressifs de territoires ». Prenons l'exemple du nord du Québec où il $\mathrm{y}$ a eu des revendications faites par les Cris de la baie James et les Naskapi de Schefferville. Les revendications portaient en partie sur la «Kaniapiskau », une région fréquentée par d'autres nations comme les innu et les Montagnais. Les Cris ont obtenu une compensation financière du gouvernement alors que les Montagnais eux n'ont pas du tout été dédommagés. Ces derniers n'ont pas voulu signer le traité, avec lequel ils n'étaient pas d'accord, parce qu'ils considéraient leur territoire original beaucoup plus vaste. Prenons l'exemple de Cartier qui au XVIe siècle trouve des Iroquoiens dans la vallée du Saint-Laurent alors que Champlain au XVIle rencontrera des Algonquiens; ou encore celui des Tuscarora, originaires de la Caroline de Sud et qui, fuyant les colons, viennent se joindre aux Cinq Nations iroquoises au cours du XVllle siècle. On le voit, le concept de territorialité ne renvoie pas nécessairement à l'idée de frontières fixes, il est beaucoup trop fluide et complexe dans le temps et dans l'espace pour ne mériter qu'une seule définition. 
On dit qu'il y a 1500 revendications territoriales en suspens et qu'une seule est réglée par année. Il y a évidemment quelque chose qui ne tourne pas rond.

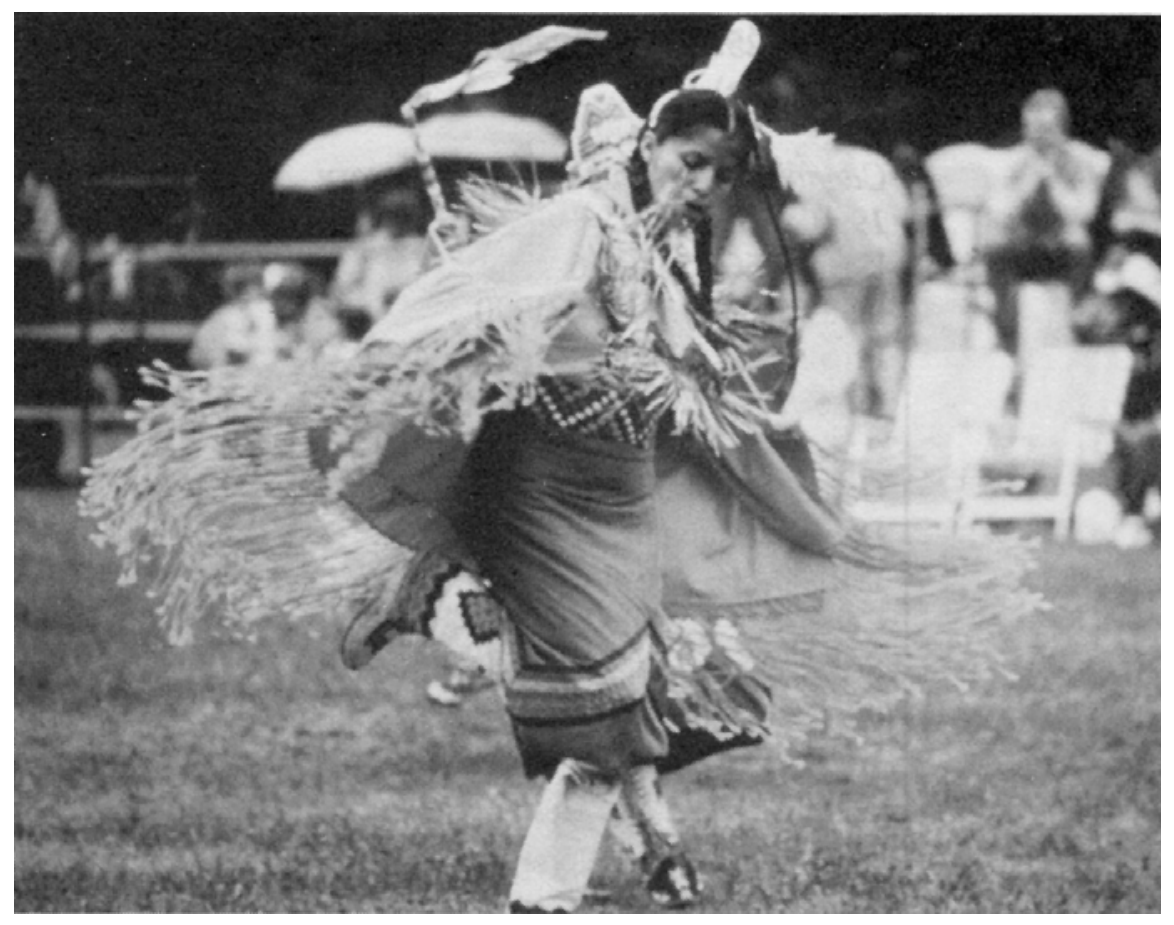

Page suivante : Amérindienne en costume traditionnel, Ontario, 1984.

Les relations entre Blancs et Amérindiens ont-elles toujours été aussi tendues? Pouvez-vous penser un exemple historique où les deux groupes se sont entendus et respectés?

Permettez-moi de vous lire un extrait des Mémoires d'outre-tombe de Chateaubriand : «Le sachem des Onondagas me reçut bien et me fit asseoir sur une natte. Il parlait anglais et entendait le français ; mon guide savait l'iroquois : la conversation fut facile ». Je me demande en lisant ces propos à quelle loi de régression, quel tracé de glissement on a obéi pour avoir avec le temps dérivé hors du champ de l'entendement. Parce que nous vivons à la fin du XXe siècle et que 
nous constatons que nos relations avec les autochtones sont tendues, nous en déduisons que certainement elles l'ont toujours été. Évidemment, il y a eu des tensions ! Les Européens apportèrent avec eux des maladies jusqu'alors inconnues en Amérique. La rougeole, la variole sont, entre autres, virtuellement responsables d'une forme de génocide. Évidemment, l'histoire a un impact sur les générations futures. Par ailleurs, des relations harmonieuses se sont quand même tissées. Prenons l'exemple des coureurs de bois et des voyageurs qui partaient de TroisRivières ou de Montréal pour aller dans la région des Grands Lacs et qui ont fini par atteindre la rivière Rouge au Manitoba, épousant des Ojibwa et des Cris. Il y avait nécessairement de bonnes relations puisque leurs enfants sont devenus ès premiers Métis. Quand les Français, au XVIle siècle traitent avec les peuples amérindiens, ils les reconnaissent comme des nations, c'est donc une relation de respect mutuel. On trouve de nombreux témoignages dans les Archives des Colonies. Ainsi, en 1701, le gouverneur de la Nouvelle-France, Callières, reçoit à Montréal des délégations amérindiennes pour échanger des captifs et créer des alliances. Le gouverneur parle d'égal à égal avec les délégués amérindiens et son discours est extrêmement éloquent. Là où les choses se détériorent vraiment, c'est au moment où l'État commence à vouloir tout gérer et policer. Il fait arpenter, il trace des frontières, il crée des réserves, et cela sans négocier avec les populations autochtones. Le XIXe siècle est véritablement le siècle de l'enfermement, et on ne peut comprendre ce qui se passe aujourd'hui sans une compréhension des événements qui sont survenus à cette époque. On prône alors la conquête de l'ouest et celle des indigènes. Le gouvernement canadien est influencé au même titre que le gouvernement américain par la doctrine du « Manifest Destiny » qui prévoit à court terme la disparition des Indiens. Pour l'indien cela signifie l'assimilation et éventuellement la disparition. C'est donc dire que la politique à l'égard des peuples autochtones se bâtit sur une fausse hypothèse : celle que les Indiens vont éventuellement disparaître. Mais comme nous le savons, ce n'est pas du tout cela qui s'est produit puisque après 1920, la population cesse de décroître. Depuis lors, elle s'est mise à augmenter à un rythme plus rapide que celui de l'ensemble de la population. Il faudrait donc complètement repenser la politique actuelle. 


\section{Que s'est-il donc passé au XIXe siècle pour qu'il y ait détérioration des rela-} tions?

La colonisation, l'établissement des colons européens jusque dans l'Ouest entraînent une politique d'enfermement qui touche les autochtones. Prenons l'exemple de l'Ontario où la population indigène était très importante. Dans la région des Grands Lacs, il y avait de nombreuses tribus qui pratiquaient l'agriculture. Avec l'arrivée des colons européens, il fallait des terres pour les fermiers, or les bonnes terres étaient souvent entre les mains des autochtones. Il a donc fallu les déterritorialiser et les enfermer dans des réserves. C'est donc de ce besoin de terres pour l'agriculture, pour l'exploitation minière que naissent les problèmes territoriaux contemporains. La situation est quelque peu différente pour la traite des fourrures où il n'y a pas eu les mêmes bouleversements, bien que les épidémies aient créé des vides dans bien des régions.

\section{Les leçons d'histoire peuvent-elles servir?}

C'est la grande question. On se dit toujours que les leçons d'histoire peuvent servir. Pourtant, dans l'affaire d'Oka, ce qui frappe d'abord, c'est l'invariabilité des revendications des Mohawk et des Algonquins depuis 1712. Voilà une leçon d'histoire que le ministère des Affaires indiennes aurait pu apprendre et qu'il n'a pas fait ! Cependant la crise d'Oka a réveille beaucoup de gens. Tout à coup, nos politiciens découvrent que les Amérindiens sont résolument sur la ligne d'horizon et qu'ils n'ont pas l'intention de disparaître. Nos intellectuels se rendent compte qu'on ne peut faire l'histoire du Canada sans faire l'histoire des autochtones. Au Québec, nous avons encore beaucoup de pain sur la planche : il faudrait au moins à Montréal, dont la population amérindienne ne cesse de grandir, un centre d'études autochtones universitaire. Avant de terminer, je voudrais dire également que nos relations avec les autochtones ne doivent pas être nécessairement tendues. Nous avons atteint l'été dernier un rare degré émotionnel auquel nous sommes peu habitués. Aujourd'hui, et les autochtones sont les premiers à le dire, il faut trouver une voie harmonieuse. C'est une sorte de défi que nous ne pouvons refuser de relever.

\section{Fin du texte}

